Publ. RIMS. Kyoto Univ.

12 Suppl. (1977), 455-464.

\title{
Zonal Spherical Functions on Some Symmetric Spaces
}

by

\author{
Jiro SEKIGUCHI*
}

\section{§ 0. Introduction}

Let $G$ be a real semisimple Lie group with finite center, and $K$ a maximal compact subgroup of $G$. A zonal spherical function on the symmetric space $X=G / K$ is an simulatneous eigenfunction $\varphi(x)$ of all the invariant differential operators on $X$ satisfying $\varphi(k x)=\varphi(x)$ for any $x \in X, k \in K$, and $\varphi(e K)=1$, where $e$ is the identity element in $G$. By the Cartan decomposition $G=K A K, \varphi(x)$ is considered as a function on $A$. And by the separation of variables, we obtain differential operators on $A$ from the invariant differential operators, which are called their radial components. In this paper, we investigate the radial components of the invariant differential operators and the zonal spherical functions when $G$ is a real, complex or quanternion unimodular group. The eigenvalues of the zonal spherical functions is parametrized by the element in $a^{*}$. Therefore, the system of differential equations on $A$ satisfied by the zonal spherical function has as many parameters as dim a. However, we can construct a new system of differential equations which admits the other parameter $\nu$. It is shown that the zonal spherical function on the real, complex or quaternion unimodular group corresponds to the case in which $\nu=\frac{1}{2}, 1,2$, respectively.

\section{$\S 1$. Radial Components of Invariant Differential Operators}

Let $\mathfrak{a}$ be a vector space of dimension $n$, and $\mathfrak{a}^{*}$ its dual space. $\mathfrak{a}^{*}$ is generated by $e_{i}, i=1,2, \cdots$, where $e_{i}(H)=t_{i}$ for $H=\left(t_{1}, \cdots, t_{n}\right)$ $\in \mathfrak{a}$.

Received September 1, 1976.

* Department af Mathematics, Faculty of Science, Kyoto University Kyoto 606, Japan. 
First we will define $n$ differential operators $\Delta_{i}^{(\nu)}, i=1,2, \cdots, n$, by the following formula,

$$
\begin{aligned}
\Delta(\zeta, \nu) & =\frac{1}{\delta(H)} \sum_{s \in \mathscr{S}_{n}}(\operatorname{det} s) e^{2 \rho(s H)} \sum_{i=1}^{n}\left(\zeta+D_{t_{s}(i)}+(n+1-2 i) \nu\right) \\
& =\zeta^{n}+\Delta_{1}^{(\nu)} \zeta^{n-1}+\Delta_{2}{ }^{(\nu)} \zeta^{n-2}+\cdots+\Delta_{n}{ }^{(\nu)} .
\end{aligned}
$$

Here

$$
\begin{aligned}
& \delta(H)=\prod_{i<j}\left(e^{t_{i}-t_{j}}-e^{t_{j}-t_{i}}\right) \\
& \rho(H)=\frac{1}{2} \sum_{i<j}\left(t_{i}-t_{j}\right) \\
& s H=\left(t_{s(1)}, \cdots, t_{s(n)}\right),
\end{aligned}
$$

for $H=\left(t_{1}, \cdots, t_{n}\right) \in \mathfrak{a}, s \in \mathfrak{S}_{n}$, and $\zeta$ is an indeterminate. For example

$$
\begin{aligned}
& \Delta_{1}^{(\nu)}=D_{t_{1}}+\cdots+D_{t_{n}} . \\
& \Delta_{2}{ }^{(\nu)}=\sum_{i<j}\left(D_{t_{i}} D_{t_{j}}-\nu \operatorname{cth}\left(t_{i}-t_{j}\right)\left(D_{t_{i}}-D_{t_{j}}\right)\right)-2\langle\rho, \rho\rangle \nu^{2} .
\end{aligned}
$$

Here $\langle$,$\rangle is the inner product on \mathrm{a}^{*}$ defined by $\left\langle e_{i}, e_{j}\right\rangle=\delta_{i j}$ :

Therem 1. The operators $\Delta_{i}{ }^{(\nu)}, i=1,2, \cdots, n$, are commutative with each other. And under the condition $\sum_{i=1}^{n} t_{i}=0$, the radial components of generators of the algebra of the invariant differential operators on symmetric spaces $\operatorname{SL}(n, \mathbb{R}) / \mathrm{SO}(n) \operatorname{SL}(n, \boldsymbol{C}) / \mathrm{SU}(n), \operatorname{SL}(n, \mathbb{H}) / \mathrm{Sp}(n)$ are given by the above operators, if we substitute $\nu$ for $\frac{1}{2}, 1,2$ respectively.

Proof. In complex unimodular group case, the radial components of invariant differential operators are known (cf. [1]). And in real unimodular group case, it is easy to compute the radial components of invariant differential operators by using a well-known formula called Cappelli's identity. In these cases, the operators $\Delta_{i}^{(\nu)}, i=1,2, \cdots, n$, are commutative, and by this fact, we can prove the commutativity of $\Delta_{i}^{(\nu)}$ for any fixed $\nu$. If we know the commutativity, it is easy to check quaternion unimodular group case.

Next, we investigate the system of differential equations 


$$
\mathscr{M}_{\lambda}^{(\nu)} ; \Delta(\zeta, \nu) u=\prod_{i=1}^{n}\left(\zeta+\lambda_{i}\right) u \text { for any } \zeta \text {. }
$$

Here $\lambda=\left(\lambda_{1}, \cdots, \lambda_{n}\right) \in \mathrm{a}^{*}$ and we assume $\sum_{i=1}^{n} \lambda_{i}=0$. This means that a solution $u$ of this system is a simultaneous eigenfunction of the differential operators $\Delta_{i}^{(\nu)}, i=1,2, \cdots, n$. Following Harish-Chandra [2], we can construct $n$ ! solutions $\Phi_{s \lambda}^{(\nu)}(H) s \in \widetilde{S}_{n}$ of this system. $\Phi_{\lambda}^{(\nu)}(H)$ is defined as follows.

$$
\Phi_{\lambda}^{(\nu)}(H)=\sum_{\mu \in L} \Gamma_{\mu}^{(\nu)}(\lambda) e^{(\lambda-2 \nu \rho-\mu)(H)},
$$

where $L=\left\{m_{1} \alpha_{1}+\cdots+m_{n-1} \alpha_{n-1} ; m_{i} \in \mathbb{N} \quad i=1,2, \cdots, n-1\right\}, \quad \alpha_{i}=e_{i}-e_{i+1}$, $N=\{0,1,2, \cdots\}$. And the coefficients $\Gamma_{\mu}^{(\nu)}(\lambda)$ satisfy the recursion formulas

$$
\sum_{s \in \subseteq_{n}}(\operatorname{det} s)\left(\prod_{i=1}^{n}\left(\zeta+\tau_{i}(\lambda-\mu, s, \nu)\right)-\prod_{i=1}^{n}\left(\zeta+\lambda_{i}\right)\right) \Gamma_{\mu+2(s \rho-\rho)}^{(\nu)}(\lambda)=0,
$$

for any $\zeta$. Here

$$
\begin{aligned}
\tau(\lambda, s, \nu) & =\lambda+2(\nu-1)(s \rho-\rho) \\
& =\left(\tau_{1}(\lambda, s, \nu), \cdots, \tau_{n}(\lambda, s, \nu)\right) .
\end{aligned}
$$

$\bar{\Phi}_{\lambda}^{\left({ }^{(\nu)}\right.}(H)$ is holomorphic in the positive Weyl chamber $C=\left\{H \in \mathfrak{a} ; \alpha_{i}(H)\right.$ $>0 i=1, \cdots, n-1\}$.

\section{$\S$ 2. An Analogue of Gegenbauer's Function in Two Variables}

In case $n=2$, the system $\mathscr{M}_{\lambda}{ }^{(\nu)}$ is well-known Gegenbauer's differential equation by taking a suitable coordinate system. In this section, we will obtain integral representation and recursion formulas for the functions satisfying the system $\mathscr{M}_{\lambda}^{(\nu)}$ in case $n=3$.

We set $\sigma_{i}=2 e_{i}-\frac{2}{3} \sum_{j=1}^{3} e_{j} i=1,2,3, x_{1}=\frac{1}{3} \sum_{i=1}^{3} e^{\sigma_{i}(H)}, x_{2}=\frac{1}{3} \sum_{i=1}^{3} e^{\sigma_{i}(-H)}$, and assume that $\sum_{i=1}^{3} e_{i}(H)=0$ for $H \in \mathfrak{a}$ in this section. we represent the operators ${\Delta_{2}}^{(\nu)}, \Delta_{3}{ }^{(\nu)}$ by $x_{1}, x_{2}$, then

$$
\begin{aligned}
\Delta_{2}^{(\nu)}= & \left(x_{2}-x_{1}^{2}\right) D_{1}^{2}+\left(1-x_{1} x_{2}\right) D_{1} D_{2} \\
& +\left(x_{1}-x_{2}\right) D_{2}^{2}-(3 \nu+1)\left(x_{1} D_{1}+x_{2} D_{2}\right)-\nu^{2} \\
\Delta_{3}^{(\nu)}= & \left(1-3 x_{1} x_{2}+2 x_{1}^{3}\right) D_{1}^{3}+3\left(x_{1}-2 x_{2}{ }^{2}+x_{1}^{2} x_{2}\right) D_{1}^{2} D_{2}
\end{aligned}
$$




$$
\begin{aligned}
& -3\left(x_{2}-2 x_{1}{ }^{2}+x_{1} x_{2}{ }^{2}\right) D_{1} D_{2}{ }^{2} \\
& -\left(1-3 x_{1} x_{2}+2 x_{2}^{3}\right) D_{2}^{3}-3(3 \nu+2)\left(\left(x_{2}-x_{1}{ }^{2}\right) D_{1}{ }^{2}-\left(x_{1}-x_{2}{ }^{2}\right) D_{1}^{2}\right) \\
& +(3 \nu+1)(3 \nu+2)\left(x_{1} D_{1}-x_{2} D_{2}\right) .
\end{aligned}
$$

First we have the following recursion formulas.

Theorem 2. There are two recursion formuras between the functions $\Phi_{\lambda}{ }^{(\nu)}(H), \lambda \in \mathfrak{a}^{*}$, if we normalize the initial value by $\Gamma_{0}{ }^{(\nu)}(\lambda)$ $=\frac{I(\lambda, \nu)}{I(2 \nu \rho, \nu)}, I(\lambda, \nu)=\prod_{i<j} B\left(\frac{\lambda_{i}-\lambda_{j}}{2}, \nu\right)(B(x, y)$ is the beta function $)$.

(*)

$$
\left\{\begin{array}{l}
3 \prod_{i<j}\left\langle\lambda, e_{i}-e_{j}\right\rangle x_{1} \Phi_{\lambda}^{(\nu)}=\sum_{k=1}^{3} \prod_{i<j}\left\langle\lambda+\nu \sigma_{k}, e_{i}-e_{j}\right\rangle \Phi_{\lambda+\sigma_{k}}^{(\nu)} \\
3 \prod_{i<j}\left\langle\lambda, e_{i}-e_{j}\right\rangle x_{2} \Phi_{\lambda}^{(\nu)}=\sum_{k=1}^{3} \prod_{i<j}\left\langle\lambda-\nu \sigma_{k}, e_{i}-e_{j}\right\rangle \Phi_{\lambda-\sigma_{k}}^{(\nu)}
\end{array}\right.
$$

Now, we consider an integral representation of a solution of the system $\mathcal{M}_{\lambda}^{(\nu)}$.

Theorem 3. Set

$$
\begin{aligned}
& G_{\nu}\left(x_{1}, x_{2} ; u_{1}, u_{2}\right)=\int_{0}^{\infty} u_{0}^{\nu-1}\left(P_{1} P_{2} P_{3}\right)^{-\nu} d u_{0} \text { for } \operatorname{Re} \nu>0, \\
& \varphi_{p_{1} p_{2}}^{(\nu)}\left(x_{1}, x_{2}\right)=c_{\nu}\left(p_{1}, p_{2}\right) \int_{0}^{\infty} \int_{0}^{\infty} u_{1}^{p_{1}-1} u_{2}^{p_{2}-1} G_{\nu}\left(x_{1}, x_{2} ; u_{1}, u_{2}\right) d u_{1} d u_{2}
\end{aligned}
$$

for $0<\operatorname{Re} p_{i}<\operatorname{Re} 2 \nu(i=1,2)$, where

$$
\begin{aligned}
& P_{i}=u_{0}+\left(1+u_{1} e^{\sigma_{i}(H)}\right)\left(1+u_{2} e^{\sigma_{i}(-H)}\right) \\
& c_{\nu}\left(p_{1}, p_{2}\right)=\frac{1}{B\left(p_{1}, 2 \nu-p_{1}\right) B\left(p_{2}, 2 \nu-p_{2}\right) B(\nu, 2 \nu)} .
\end{aligned}
$$

Then $\varphi_{p_{1} p_{2}}^{(\nu)}(x)$ has following properties.

(1) $\varphi_{p_{1} p_{2}}^{(\nu)}$ is a solution of the system $\mathscr{H}_{\lambda}^{(\nu)}$, where

$$
p_{1}=\frac{\lambda_{2}-\lambda_{1}+2 \nu}{2}, p_{2}=\frac{\lambda_{3}-\lambda_{2}+2 \nu}{2} .
$$

(2) $\varphi_{p_{1} p_{2}}^{(\nu)}(1,1)=1$ 
(3) (Generating function.)

$$
G_{\nu}(x, u)=\sum_{m, n=1}^{\infty} \frac{(2 \nu, m)(2 \nu, n)}{m ! n !}(-1)^{m+n} \varphi_{m n}^{(\nu)}(x) u_{1}{ }^{m} u_{2}{ }^{n} .
$$

(4) (Functional equation.)

$$
\varphi_{2 \nu-p_{1}, p_{1}+p_{2}-\nu}^{(\nu)}=\varphi_{p_{1}+p_{2}-\nu, 2 \nu-p_{2}}^{(\nu)}=\varphi_{p_{1} p_{2}}^{(\nu)} .
$$

(5) There is a relation between $\varphi_{p_{1} p_{2}}^{(\nu)}$ and $\Phi_{s \lambda}^{(\nu)}, s \in \widetilde{S}_{3}$.

$$
\varphi_{p_{1} p_{2}}^{(\nu)}(x)=\sum_{s \in \mathbb{S}_{3}} \Phi_{s \lambda}^{(\nu)}(H) \text { for } H \in C .
$$

(6) The recursion formulas $\left(^{*}\right)$ are also valid if we change $\Phi_{\lambda}^{(\nu)}$ by $\varphi_{p_{1} p_{2}}^{(\nu)}$.

The function $\varphi_{p_{1} p_{2}}^{(\nu)}(x)$ is an analogue of Gegenbauer's function in two variables. $\varphi_{p_{1} p_{2}}^{(\nu)}$ satisfies many interesting properties including (1) $\sim(6)$ in Theorem 3. And if we substitute $\nu$ for $\frac{1}{2}, 1,2, \varphi_{p_{1} p_{2}}^{(\nu)}$ is a zonal spherical function on $\mathrm{SL}(3, \mathbb{R}) / \mathrm{SO}(3), \operatorname{SL}(3, \boldsymbol{C}) / \mathrm{SU}(3), \operatorname{SL}(3, \mathbb{H})$ /Sp(3), respectively.

Remark. After the work, I knew that Prof. Koornwinder ([3]) has obtained the differential operators $\Delta_{2}^{(\nu)}, \Delta_{3}^{(\nu)}$, and investigated the orthogonal polynomials $\varphi_{m n}^{(\nu)}(x) \quad(m, n \in \mathbb{N})$.

\section{References}

[1] Gel'fand, I. M. and Naimark, M. A., Unitäre darstellungen der klassischen Gruppen, Akademie Verlag, 1957.

[2] Harish-Chandra, Spherical functions on a semisimple Lie group I, Amer. J. Math., 80 (1958), 241-310.

[3] Koornwinder, T. H., Orthogonal polynomials in two variables which are eigenfunctions of two algebraically independent partial differential operators, III, IV, Jndag. Math., 35 (1974), 357-381. 
